\title{
Mean-Variance Utility
}

\author{
Yutaka Nakamura \\ University of Tsukuba \\ Graduate School of Systems and Information Engineering \\ Division of Social Systems and Management \\ 1-1-1 Tennnoudai, Tsukuba, Ibaraki 305-8573, Japan \\ Tel.: +81-(0)29-853-5547; Fax: +81-(0)29-853-5070 \\ E-mail address: nakamura@sk.tsukuba.ac.jp
}

September 21, 2015

\begin{abstract}
The mean-variance utility postulates that random variables with the same mean and variance should be equally desirable. This paper presents and discusses necessary and sufficient preference-based axioms for the existence of mean-variance utility in which any condition on variances but mean-values is not explicitly specified. Furthermore, we investigate necessary and sufficient axioms for four types of additively separable forms of mean and variance in the utility representation.
\end{abstract}

Keywords: Mean-variance analysis, mean-variance utility, decision making under risk, constant risk aversion

JEL Classification: D81 


\section{Introduction}

Expected utility (EU) maximization and mean-variance (MV) analysis are two tenets in rational decision making under risk. A decision criterion in MV analysis is maximization of MV utility in which random variables with the same mean and variance should be equally desirable. It has been argued that EU maximization is incompatible with MV criterion unless von Neumann-Morgenstern utility function is given by a quadratic function or random variables under consideration are appropriately restricted (e.g., see Chapter 4 in [1]).

Preference structures of economic agents who maximize expected utility are well known (e.g., see [2, 3]) since numerous axiomatic refinements and generalizations have been investigated under various structural assumptions to remedy the pioneering works by von Neumann and Morgenstern [20] and Savage [19]. On the other hand, little is known for MV utility although it has been widely used in finance as an alternative to EU maximization since the pioneering work by Markowitz $[13,14]$.

To the best of my knowledge, Pollatsek and Tversky [16] is the first to present conditions for the existence of MV utility which is represented by the weighted sum of mean-value and variance. Their axiomatization is preference-based, but their continuity condition is explicitly stated in terms of means and variances of random variables under consideration. The next challenge I am aware of is due to Fishburn [8, 9]. Assuming equal desirability of random variables with the same mean and variance, he showed that a condition, dubbed zero-degree stochastic dominance, implies that preferences are represented by a lexicographic MV model in which random variables with larger means are more preferable, while random variables with larger variances are less preferable as long as their mean-values are the same.

For the general functional form of MV utility which is strictly increasing in mean-values and (strictly) decreasing in variances, Epstein [6] characterized its existence under Fréchet differentiability and some other technical conditions. He also provided a condition, dubbed constant risk aversion, which is necessary and sufficient for Pollatsek-Tversky model to hold as a special case of his general MV utility. As a different approach to arrive at MV utility, Löffler [12] proved under a security market framework that so-called strict variance aversion (see [4]) together with strict monotonicity implies that a trader's preferences obey maximization of MV utility.

The aim of the paper is to present an alternative characterization of MV utility. Unlike Löfler's framework, we study preference conditions for random variables on the real line as traditional EU axiomatizations. Unlike Epstein's characterization, we do not assume the existence of a functional $U$ on a set $\mathbb{Y}$ of random variables which represents the preferences. Our axiomatization of the preferences provides a constructive way of MV utility on $\mathbb{Y}$. Furthermore, it is different from Pollatsek-Tversky's axiomatization and Fishburn's investigation in that our axioms do not explicitly assume any knowledge of variances. While Epstein [6] introduced a general notion of decreasing risk aversion which makes Fréchet differentiable $U$ depend only on mean and variance, our crucial axiom specifies the exchange preferences between two simple random variables $X$ and $Y$, in which, given a binary 50-50 fair random variable $Z$, it is asked which of the two compound random variables is preferred; one is to add $X$ to the positive outcome of $Z$ and $Y$ to the negative outcome, and the other is to exchange 
additions of $X$ and $Y$ to outcomes of $Z$. The notion of exchange preferences between a fair random variable and sure outcome 0 is known as downside risk aversion (e.g., see Chapter 2 in [5]). Also, its more general form, called constant exchange risk property in [7], was applied to specify functional forms of von Neumann and Morgenstern utility functions.

We also investigate necessary and sufficient axioms for four types of additively separable forms of mean and variance in the MV utility representation. The most general of these is given by sum of a strictly increasing function $f$ of mean-values and a strictly decreasing function $g$ of variances. An adaptation of Thomsen condition well known in additive conjoint measurement (see [11]) to the present framework is shown to be necessary and sufficient for the separation. The other three types of additive separabilities are obtained by variants of constant risk aversion. We derive the first of these, which makes $f$ linear, by applying the well known property of constant absolute risk aversion. Together with constant absolute risk aversion, we apply the another well known property, constant proportional (also, called relative) risk aversion, to obtain the third type of additive separable form in which $g$ becomes a square root function, i.e., standard deviations. The last type is Pollatsek-Tversky model. We prove that the model follows by applying Epstein's constant risk aversion to the present context. While constancies of absolute and proportional risk aversions come from the property that agent's attitudes toward risk are unaffected by changing levels of the agent's wealth, Epstein's constancy is more demanding in that the agent wealth levels can be changed to any random variables.

Recently, Qu [17] studied a complementary approach to mean-variance preferences in decision making under uncertainty. He adopted the Anscombe-Aumann framework and derived two additively separable representations. However, his approach assumes the first-order stochastic dominance which is not necessarily compatible with mean-variance preferences (see [9]), and does not allow for the general non-separable representation.

The paper is organized as follows. Section 2 introduces three axiom systems for the existence of MV utility. The first one is concerned with simple random variables. The second covers the set of all random variables with bounded supports. The third focusses on the set of random variables with finite means which are bounded in preference. In Section 3 , we presents necessary and sufficient axioms for four types of additively separable forms of mean and variance. Section 4 concludes the paper. All the sufficiency proofs of the MV representation theorems in Sections 2 and 3 are gathered respectively in Appendices A and B.

\section{Mean-Variance Utility}

\subsection{Preliminary Definitions}

Let $\mathbb{X}$ be the set of all random variables defined on a state space $S$ whose cumulative distribution functions are right-continuous on $\mathbb{R}$. Elements of $\mathbb{X}$ will be denoted by capital letters, $X, Y, Z$, and so on. Assume that a binary preference relation $\precsim$ is given on $\mathbb{X}$. Then $X \precsim Y$ reads as "random variable $X$ is not preferred to random variable $Y$." Asymmetric 
and symmetric parts of $\precsim$, denoted $\prec$ and $\sim$ respectively, are defined in the usual way as follows: for all $X, Y \in \mathbb{X}, X \prec Y$ if $\neg(Y \precsim X)$, and $X \sim Y$ if $X \precsim Y$ and $Y \precsim X$.

The probability density function and cumulative distribution function of $X$ are respectively denoted by $p_{X}$ and $P_{X}$. Let $N$ be the set of all positive integers. When $X$ is a random variable with finite support $\left\{x_{1}, \ldots, x_{n}\right\} \subset \mathbb{R}$ for some $n \in N$, where $x_{1}<x_{2}<\cdots<x_{n}$, probability with which wealth level $x_{k}$ obtains under $X$ is given by $p_{X}\left(\left\{x_{k}\right\}\right)=P_{X}\left(x_{k}\right)-$ $P_{X}\left(x_{k-1}\right)$ for $k=1, \ldots, n$, where $x_{0}$ is any number such that $P_{X}\left(x_{0}\right)=0$ and $x_{0}<x_{1}$. In what follows, we shall write $p_{X}\left(x_{k}\right)$ in place of $p_{X}\left(\left\{x_{k}\right\}\right)$.

Given $n$ random variables $X_{1}, \ldots, X_{n}$ and numbers $\lambda_{1}, \ldots, \lambda_{n} \in(0,1)$ with $\sum_{i=1}^{n} \lambda_{i}=1$, we define a new random variable $X$, called $\left(\lambda_{1}, \ldots, \lambda_{n-1}\right)$-compound of $X_{1}, \ldots, X_{n}$, to satisfy that $p_{X}(I)=\sum_{i=1}^{n} \lambda_{i} p_{X_{i}}(I)$ for all intervals $I \subseteq \mathbb{R}$. When the compound probabilities $\lambda_{1}, \ldots, \lambda_{n}$ are not specified at the outset, we simply says that $X$ is a compound of $X_{1}, \ldots, X_{n}$. In particular, $\lambda$-compound of random variables $X$ and $Y$ will be denoted by $\langle X ; \lambda ; Y\rangle$. Each $x$ will be identified with a random variable which gives wealth level $x$ with probability one. For $X \in \mathbb{X}$ and $a \in \mathbb{R}$, let $X+a$ and $a X$ (dubbed a-proportional fraction of $X$ ) be random variables whose cumulative distribution functions are given by $P_{X+a}(x+a)=P_{X}(x)$ and $P_{a X}(a x)=P_{X}(x)$ for all $x \in \mathbb{R}$. In particular, when $a=-1, a X$, denoted by $-X$, is a mirror image of $X$. A random variable $X$ is said to be symmetric if $X=-X$. Note that symmetric random variables are fair, i.e., their mean-values equal 0.

Let $\mathbb{R}_{+}$denote the half line of nonnegative numbers. Let $\mu_{X}$ and $\sigma_{X}^{2}$ respectively denote the mean-value and variance of random variable $X$ if they exist, so $\sigma_{X}$ is the standard deviation of $X$. Consider a subset $\mathbb{Y}$ of $\mathbb{X}$, whose elements have finite mean-values and variances. We say that ( $\mathbb{Y}, \precsim)$ has an $M V$ utility function $U$ if it is a real valued function on $\mathbb{R} \times \mathbb{R}_{+}$such that, for all $X, Y \in \mathbb{Y}$,

$$
X \precsim Y \Longleftrightarrow U\left(\mu_{X}, \sigma_{X}^{2}\right) \leq U\left(\mu_{Y}, \sigma_{Y}^{2}\right)
$$

where $U$ is strictly increasing in the first argument and strictly decreasing in the second.

\subsection{Necessary and Sufficient Axioms}

Depending on structures of $\mathbb{Y} \subseteq \mathbb{X}$, we shall introduce three axiom systems which are necessary and sufficient for $(\mathbb{Y}, \precsim)$ to have an MV utility function. For each $a \in \mathbb{R}$ and $\mathbb{Y} \subseteq \mathbb{X}$, let $\mathbb{Y}_{a}$ denote the set of all random variables in $\mathbb{Y}$ whose mean-values equal $a$. In what follows, we shall assume that $\mathbb{Y} \subseteq \cup_{a} \mathbb{X}_{a}$, i.e., $\mathbb{Y}$ is a set of random variables whose mean-values are finite.

We shall consider three types of random variables. The first type is concerned with random variables with finite supports, which will be called simple, but otherwise non-simple. Cumulative distribution functions of simple random variables are step-functions with finite steps. The second type consists of all random variables whose supports are bounded. Of course, simple random variables have bounded supports and there is a non-simple random variable with a bounded support. 
We say that a random variable $X$ with finite mean-value is bounded in preference if $Y \precsim X$ for some simple random variable $Y$ with $\mu_{Y}=\mu_{X}$. The third type consists of all random variables which are bounded in preference. In our axiomatization, it is easily shown that those random variables have finite variances. By Theorem 2 below, it will be guaranteed that random variables with bounded supports are bounded in preference.

The first axiom system for $(\mathbb{Y}, \prec)$, in which $\mathbb{Y}$ will be assumed to be the set of all simple random variables, is necessary and sufficient for the existence of an MV utility function for all simple random variables. It consists of the following seven axioms, which are understood as applying to all $X, Y, Z \in \mathbb{Y}$ and all $a, b \in \mathbb{R}$.

Axiom A1. $\precsim$ on $\mathbb{Y}$ is a weak order.

Axiom A2. If $X, Y, Z \in \mathbb{Y}_{a}$ and $X \prec Y$, then $\langle X ; \lambda ; Z\rangle \prec\langle Y ; \lambda ; Z\rangle$ for all $0<\lambda<1$.

Axiom A3. If $X, Y, Z \in \mathbb{Y}_{a}, X \prec Z$, and $Z \prec Y$, then $\langle X ; \alpha ; Y\rangle \prec Z$ for some $\alpha \in(0,1)$.

Axiom A4. If $X \in \mathbb{Y}_{0}$, then $X+a \sim-X+a$.

Axiom A5. If $X$ and $Y$ are simple, and $a>0$, then

$$
\mu_{X} \leq \mu_{Y} \Longleftrightarrow\left\langle X-a ; \frac{1}{2} ; Y+a\right\rangle \precsim\left\langle Y-a ; \frac{1}{2} ; X+a\right\rangle .
$$

Axiom A6. If $X$ is simple, then $X \sim c$ for some $c \in \mathbb{R}$.

Axiom A7. If $X$ is simple and fair, and $a<b$, then $X+a \prec X+b$.

First of all, we note that the above seven axioms apply to different sets of random variables. The first four axioms apply to $\mathbb{Y}$ or sets of random variables in $\mathbb{Y}$ with the same mean-values. Of course, specifications of $\mathbb{Y}$ depend on the axiom systems which we shall propose. The last three axioms apply to the set of simple random variables or the set of fair simple random variables, so that they are common to all three axiom systems.

Illustration of the axioms are given in order. The first three axioms are restricted versions of the standard axioms of EU maximization (e.g., see [10]). In Axiom A1, which unrestrictedly applies to all random variables in $\mathbb{Y}$, a weakly ordered $\precsim$ means that it is complete (i.e., for all $X, Y \in \mathbb{Y}, X \precsim Y$ or $Y \precsim X$ ) and transitive (i.e., for all $X, Y, Z \in \mathbb{Y}$, if $X \precsim Y$ and $Y \precsim Z$, then $X \precsim Z$ ). Axioms A2 and A3 restrictedly apply to all random variables in $\mathbb{Y}$ with the same mean-value. Axiom A2 is the independence condition. Axiom A3 is a weak version of the continuity condition, dubbed Lower Archimedean axiom in [15], who proved in a more general setup that Axioms A1-A3 restricted to $\mathbb{Y}_{a}$ are necessary and sufficient for the existence of a linear functional $U_{a}$ on $\mathbb{Y}_{a}$ such that, for all $X, Y \in \mathbb{Y}_{a}$ and all $0<\lambda<1$, $X \precsim Y$ iff $U_{a}(X) \leq U_{a}(Y)$, and $U_{a}(\langle X ; \lambda ; Y\rangle)=\lambda U_{a}(X)+(1-\lambda) U_{a}(Y)$. Furthermore, in our present framework, it will be shown later in Lemma A3 of Appendix A that there is a real valued function $u_{a}$ on $\mathbb{R}$ such that, for all simple $X$ with $\mu_{X}=a, U_{a}(X)=\sum p_{X}(x) u_{a}(x)$. 
Axioms A4 and A5 are typical of MV utility. Axiom 4 says that any fair random variable in $\mathbb{Y}_{0}$ is indifferent to its mirror image at any wealth level. To illustrate Axiom 5, consider a situation that, given a binary 50-50 fair random variable $Z=\left\langle-a ; \frac{1}{2} ; a\right\rangle$ with $\left.a\right\rangle 0$, it is asked which of the two compound random variables is preferred; one is to add $X$ to the positive outcome of $Z$ and $Y$ to the negative outcome, and the other is to exchange additions of $X$ and $Y$ to outcomes of $Z$. Axiom A5 requires that the former is at least as preferable as the latter if and only if mean-value of $X$ is not greater than mean-value of $Y$. Although mean-values of the compound random variables are equal, it is easily shown that variance of the former is not greater than variance of the latter. Axiom A5 is a slight modification of the constant exchange risk property with $\frac{1}{2}$ the exchange probability proposed in [7], in which it was shown that, under EU framework, von Neumann-Morgenstren utility functions must be quadratic. In our framework, Axiom A5 together with Axiom A4 implies that each $u_{a}$ is symmetric around $a$ and quadratic, so that $U_{a}(X)=-\sigma_{X}^{2}$ for all simple $X$ with $\mu_{X}=a$.

The remaining two axioms combine all $u_{a}$ to construct an MV utility function $U$. Axiom 6 requires that any simple random variable has its certainty equivalent, which will be used to define the $U$ value at $\left(\mu_{X}, \sigma_{X}^{2}\right) \in \mathbb{R} \times \mathbb{R}_{+}$. Axiom A7 simply requires monotonicity of $U$ with respect to mean-values.

The representational implication of Axioms A1-A7 for simple random variables is stated as follows.

Theorem 1. Suppose that $\mathbb{Y}$ is the set of all simple random variables. Then Axioms A1-A7 hold for ( $\mathbb{Y}, \precsim)$ if and only if $(\mathbb{Y}, \precsim)$ has an $M V$ utility function.

While necessity of the axioms easily follows, a sufficiency proof will be deferred to Appendix A.

The second axiom system for ( $\mathbb{Y}, \precsim)$ is concerned with random variables with bounded supports. It assumes that Axioms A1-A7 hold when $\mathbb{Y}$ is the set of all random variables whose supports are bounded. Therefore, it follows from the similar arguments of Axioms A1-A3 for the first axiom system that, for every $a \in \mathbb{R}$, there is an order-preserving linear functional $U_{a}$ on the set of all random variables whose mean-values equal $a$ and supports are bounded. In particular, $U_{a}(X)=-\sigma_{X}^{2}$ for all simple $X$ with $\mu_{X}=a$. Thus, our task is to guarantee that $U_{a}(X+a)=-\sigma_{X}^{2}$ for all symmetric and non-simple $X$ with bounded supports. To see this, take any non-simple $X$ with a bounded support and $\mu_{X}=a$. It follows from linearity of $U_{a}$ that

$$
2 U_{a}\left(\left\langle X-a ; \frac{1}{2} ;-X+a\right\rangle+a\right)=U_{a}(X)+U_{a}(-X+2 a) .
$$

Since $\mu_{X-a}=0$, Axiom A4 implies that $X=(X-a)+a \sim-(X-a)+a=-X+2 a$, so that $U_{a}(X)=U_{a}(-X+2 a)$. Hence, $U_{a}\left(\left\langle X-a ; \frac{1}{2} ;-X+a\right\rangle+a\right)=U_{a}(X)$. Since $\left\langle X-a ; \frac{1}{2} ;-X+a\right\rangle$ is symmetric and non-simple, it suffices to show that $U_{a}(Y+a)=-\sigma_{Y}^{2}$ for all symmetric and non-simple $Y$ with bounded supports.

To accomplish the task for a symmetric and non-simple $X$ with a bounded support, we shall approximate the cumulative distribution function $P_{X}$ on the negative domain $\mathbb{R}_{-}$, the half line of nonpositive numbers, from two directions. One is from below by a sequence 
of simple symmetric random variables, which is increasing in the sense of mean-preserving spread, and the other is from above by a sequence of simple symmetric random variables, which is decreasing in the sense of mean-preserving contraction. Because of symmetry, the former (respectively, the latter) sequence decreasingly (respectively, increasingly) approaches to $P_{X}$ on the positive domain $\mathbb{R}_{+}$.

We shall impose the following dominance axiom for the aforementioned approximation to work.

Axiom A8. For all symmetric and non-simple random variables $X$ and $Y$ with bounded supports, if $p_{X}([a, \infty)) \leq p_{Y}([a, \infty))$ for all $a>0$, then $Y+x \precsim X+x$ for all $x \in \mathbb{R}$.

This axiom says that, given symmetric and non-simple random variables $X$ and $Y$ with bounded supports, if $Y$ is a mean-preserving spread of $X$ (or, in other words, $X$ is a meanpreserving contraction of $Y$ ), then $Y$ should not be preferred to $X$ at all wealth level.

The representational implication of Axioms A1-A8 for random variables with bounded supports is stated as follows.

Theorem 2. Suppose that $\mathbb{Y}$ is the set of all random variables with bounded supports. Then Axiom A1-A8 hold for ( $\mathbb{Y}, \precsim)$ if and only if ( $\mathbb{Y}, \precsim)$ has an MV utility function.

It is easy to see that the axioms are necessary for the representation. The sufficiency proof will be deferred to Appendix A.

The third axiom system for ( $\mathbb{Y}, \precsim)$ focuses on the set of all random variables which are bounded in preference, so that infinite variances will be excluded. It assumes that Axioms A1-A8 hold when $\mathbb{Y}$ is the set of all bounded-in-preference random variable. It is easy to see by Axiom 8 that all random variables with bounded supports are bounded in preference, and there is a bounded-in-preference random variable with an unbounded support. Again from the similar arguments of Axioms A1-A3, for every $a \in \mathbb{R}$, we know the existence of an order-preserving linear functional $U_{a}$ on the set of all bounded-in-preference random variables whose mean values equal $a$ such that $U_{a}(X)=-\sigma_{X}^{2}$ for all random variables $X$ with bounded supports and $\mu_{X}=a$. Thus our task is again to prove that $U_{a}(X)=-\sigma_{X}^{2}$ for all bounded-in-preference random variables $X$ with unbounded supports and $\mu_{X}=a$. But it follows from the similar argument to the second axiom system that it suffices to show that, for all $a \in \mathbb{R}, U_{a}(X+a)=-\sigma_{X}^{2}$ for all symmetric bounded-in-preference $X$ with unbounded supports.

To accomplish the task for a symmetric bounded-in-preference $X$ with unbounded supports, we shall introduce a symmetric truncation of $X$ as follows. Since $X$ is symmetric and its support is unbounded, probability masses $p_{X}([a, \infty))$ and $p_{X}((-\infty, a])$ do not vanish for all $a>0$. By a truncation of $X$ at $a>0$, it means a random variable, denoted $X_{a, \infty}$, for which probability masses $p_{X}([a, \infty))$ and $p_{X}((-\infty, a])$ are respectively transferred to points $a$ and $-a$, i.e., 


$$
\begin{aligned}
p_{X_{a, \infty}}(a) & =p_{X}([a, \infty)), \\
p_{X_{a, \infty}}(-a) & =p_{X}((-\infty,-a]), \\
p_{X_{a, \infty}}(I) & =p_{X}(I) \text { for all intervals } I \subseteq(-a, a) .
\end{aligned}
$$

Obviously, $X_{a, \infty}$ is a symmetric random variable with a bounded support. Furthermore, $X_{a, \infty}$ is a mean-preserving contraction of $X$ and $X_{a, \infty}$ is a mean-preserving spread of $X_{b, \infty}$ whenever $a>b$. Now we approximate the cumulative distribution function $P_{X}$ on the positive domain $\mathbb{R}_{+}$from above only. The approximation is accomplished by a sequence of symmetric truncations of $X$, which is increasing in the sense of mean-preserving spread.

For the approximation to work, we shall impose the following truncation continuity axiom.

Axiom A9. For all symmetric and bounded-in-preference random variables $X$ and $Y$ with unbounded supports, and for all $x \in \mathbb{R}$, if $X \prec Y$, then $X_{a, \infty}+x \prec Y+x$ for some $a>0$.

This axiom says that, given symmetric bounded-in-preference random variables $X$ and $Y$ whose supports are unbounded, if $Y$ is preferred to $X$, then, at any wealth level, $Y$ is still preferred to a symmetric truncation of $X$ at some $a>0$.

The representational implication of Axioms A1-A9 for bounded-in-preference random variables is stated as follows.

Theorem 3. Suppose that $\mathbb{Y}$ is the set of all bounded-in-preference random variables. Then Axioms A1-A9 for ( $\mathbb{Y}, \precsim)$ hold if and only if ( $\mathbb{Y}, \precsim)$ has an $M V$ utility function.

Again, the necessity of Axiom A9 is easily verified. The sufficiency proof will be deferred to Appendix A.

\section{Additively Separable Forms}

This section studies additively separable effects of mean-values and variances on the utility values of random variables. Specific separable functional forms of an MV utility function $U\left(\mu_{X}, \sigma_{X}^{2}\right)$ that we shall examine are given as follows:

(a) additive trade-off: $U\left(\mu_{X}, \sigma_{X}^{2}\right)=f\left(\mu_{X}\right)-g\left(\sigma_{X}^{2}\right)$ for a strictly increasing continuous and unbounded function $f$ on $\mathbb{R}$ and a strictly increasing continuous function $g$ on $\mathbb{R}_{+}$;

(b) additive mean-dispersion: $U\left(\mu_{X}, \sigma_{X}^{2}\right)=\mu_{X}-g\left(\sigma_{X}^{2}\right)$ for a strictly increasing continuous function $g$ on $\mathbb{R}_{+}$;

(c) additive mean and standard deviation: $U\left(\mu_{X}, \sigma_{X}^{2}\right)=\mu_{X}-\theta \sigma_{X}$ for a $\theta>0$.

(d) additive mean-variance: $U\left(\mu_{X}, \sigma_{X}^{2}\right)=\mu_{X}-\theta \sigma_{X}^{2}$ for a $\theta>0$.

Since MV utility functions derived from Axioms A1-A7 may not be continuous on $\mathbb{R} \times$ $\mathbb{R}_{+}$, those axioms are not sufficiently rich enough to guarantee exact trade-offs between $f$ 
evaluation of mean-values and $g$ evaluation of variances in model (a). Therefore, we need to strengthen two continuity axioms, Axioms A3 and A4, by the following two axioms:

Axiom A3*. If $X, Y \in \mathbb{Y}_{a}, Z \in \mathbb{Y}, X \prec Z$, and $Z \prec Y$, then $\langle X ; \alpha ; Y\rangle \prec Z$ for some $\alpha \in(0,1)$.

Axiom A6*. If $X$ and $Y$ are simple, then $X \sim Y+c$ for some $c \in \mathbb{R}$.

While, in Axiom A3, $Z$ was assumed to belong to $\mathbb{Y}_{a}$ as other two random variables $X$ and $Y$ do, Axiom $\mathrm{A}^{*}{ }^{*}$ requires that $Z$ be any in $\mathbb{Y}$ to evaluate trade-offs along with varying variances between random variables with different mean-values. What Axiom A6 required was exact trade-offs between any simple random variable and outcome. Axiom A6* is more demanding in that exact trade-offs along with varying mean-values should be made between simple random variables with different variances.

Now we study conditions for models (a)-(d) when $\mathbb{Y}$ is the set of all simple random variables. First of all, we shall consider additive trade-off (a), in which MV utility function is simply the sum of two functions of mean-values and variances. This functional form is obtained by applying additive conjoint measurement (see [11]). Since $U$ is two-dimensional, the crucial condition for additive separability (a) is the following, well known as Thomsen condition.

Axiom B1 (Thomsen condition). For all simple and fair random variables $X, Y$, and $Z$ and all $a, x, y \in \mathbb{R}$, if $X+a \sim Z+a+x$, and $Y+a \sim Z+a+y$, then $X+a+y \sim Y+a+x$.

This axiom is also called a cancellation. To see this, we assume from two premises of the axiom that $U\left(\mu_{X}+a, \sigma_{X}^{2}\right)=U\left(\mu_{Z}+a+x, \sigma_{Z}^{2}\right)$ and $U\left(\mu_{Z}+a+y, \sigma_{Z}^{2}\right)=U\left(\mu_{Y}+a, \sigma_{Y}^{2}\right)$. Noting that $\mu_{X}=\mu_{Y}=\mu_{Z}=0$, if additive trade-off (a) is to hold, then

$$
\begin{aligned}
f(a)-g\left(\sigma_{X}^{2}\right) & =f(a+x)-g\left(\sigma_{Z}^{2}\right), \\
f(a+y)-g\left(\sigma_{Z}^{2}\right) & =f(a)-g\left(\sigma_{Y}^{2}\right) .
\end{aligned}
$$

Adding these two together cancels out $Z$-related terms to give $f(a+y)-g\left(\sigma_{X}^{2}\right)=f(a+x)-$ $g\left(\sigma_{Y}^{2}\right)$. Hence, $U\left(\mu_{X}+a+y, \sigma_{X}^{2}\right)=U\left(\mu_{Y}+a+x, \sigma_{Y}^{2}\right)$, which is $X+a+y \sim Y+a+x$.

Axiomatizations of the remaining three separable forms are respectively based on three types of attitudes toward risk that are invariant (or constant) over any changes of agent's wealth levels. Model (b) clearly satisfies the following standard concept of constant absolute risk aversion, which says that if holding a fair and simple random variable $X$ at wealth level $w$ is indifferent to receiving $a>0$ (or paying $-a$ if $a<0$ ) at $w$, then so is at any wealth level.

Axiom B2 (Constant absolute risk aversion). For all simple and fair random variables $X$, if $X+w \sim a+w$ for some $w, a \in \mathbb{R}$, then $X+x \sim a+x$ for all $x \in \mathbb{R}$. 
It will be shown that this condition implies Thomsen condition, so that additive trade-off model obtains. Furthermore, it makes mean-values linearly contribute to the whole utility values.

Safra and Segal [18] studied effects of combination of constant absolute and constant proportional risk aversions on various functional forms which are assumed to represent preferences. They termed the combination of the two constant risk aversion, formerly stated as follows.

Axiom B3 (Constant risk aversion). For all simple and fair random variables $X$, if $X+w \sim a+w$ for some $w, a \in \mathbb{R}$, then $\lambda X+x \sim \lambda a+x$ for all $x \in \mathbb{R}$ and all $\lambda>0$.

This axiom says that if holding a fair and simple random variable $X$ at wealth level $w$ is indifferent to receiving $a>0$ (or paying $-a$ if $a<0$ ) at $w$, then any $\lambda$-proportional fraction of $X$ will be judged to be indifferent to receiving $\lambda a>0$ (or paying $-\lambda a$ if $a<0$ ) at any wealth level. Together with constant absolute risk aversion (i.e., $\lambda=1$ ), it is proved that in additive mean-dispersion model (b), $g$ function of variances becomes a square root function, i.e., it yields standard deviations.

Additive mean-variance model (d) was first axiomatized by Pollatsek and Tversky [16]. Later, Epstein [6] derived the model under the assumption that utility functional for random variables is Fréchet differentiable. His necessary and sufficient condition may be stated in our framework as follows, where, for all simple random variables $X$ and $Y$, we define $X+Y$ to be a random variable which yields outcome $x+y$ with probability $p_{X}(x) p_{Y}(y)$.

Axiom B4 (Constant risk aversion for random wealth changes). For all simple random variables $X$ and $Y$, if $X+Y \sim Y$, then $X+Z \sim Z$ for all simple random variables $Z$.

While constancies of absolute and proportional risk aversions come from the requirement that agent's attitudes toward risk are unaffected by changing levels of the wealth, Epstein's constancy is more demanding in that the agent's wealth levels can be changed to any random variables. This axiom is interpreted as follows: if holding a simple random variable $X$ is indifferent to the status quo when the decision maker's wealth level is given by a random variable $Y$, which is statistically independent of $X$, then the situation does not change although his wealth level is transferred to any random variable as long as it remains to be statistically independent of $X$.

In our framework, Axiom B4 is also necessary and sufficient for model (d). To grasp the idea, recall that, for all simple random variables $X$ and $Y, \mu_{X+Y}=\mu_{X}+\mu_{Y}$ and $\sigma_{X+Y}^{2}=\sigma_{X}^{2}+\sigma_{Y}^{2}$. Thus, on the $\left(\mu_{X}, \sigma_{X}^{2}\right)$-plane, Axiom B4 requires that if point $(a, b)+(c, d)$ is indifferent to point $(a, b)$, i.e., $U(a+c, b+d)=U(a, b)$, then $(x, y)+(c, d)$ is indifferent to $(x, y)$ for all $(x, y) \in \mathbb{R} \times \mathbb{R}_{+}$. Hence indifferent curves on the $\left(\mu_{X}, \sigma_{X}^{2}\right)$-plane is the straight lines parallel to the vector $(c, d)$, so model $(\mathrm{d})$ follows.

Additively separable representations of $U$ are summarized in the following theorem.

Theorem 4. Let $\mathbb{Y}$ be the set of all simple random variables. Suppose that Axioms A1, A2, A3*, A4, A5, A6*, and A7 hold for ( $\mathbb{Y}, \precsim)$. Then we have: 
(1) Axiom B1 holds if and only if $U\left(\mu_{X}, \sigma_{X}^{2}\right)=f\left(\mu_{X}\right)-g\left(\sigma_{X}^{2}\right)$ for all simple random variables $X$ and for a strictly increasing, continuous, and unbounded function $f$ on $\mathbb{R}$ and a strictly increasing and continuous functions $g$ on $\mathbb{R}_{+}$;

(2) Axiom B2 holds if and only if $U\left(\mu_{X}, \sigma_{X}^{2}\right)=\mu_{X}-g\left(\sigma_{X}^{2}\right)$ for all simple random variables $X$ and a strictly increasing and continuous function $g$ on $\mathbb{R}_{+}$;

(3) Axiom B3 holds if and only if $U\left(\mu_{X}, \sigma_{X}^{2}\right)=\mu_{X}-\theta \sigma_{X}$ for all simple random variables $X$ and some $\theta>0$.

(4) Axiom B4 holds if and only if $U\left(\mu_{X}, \sigma_{X}^{2}\right)=\mu_{X}-\theta \sigma_{X}^{2}$ for all simple random variables $X$ and some $\theta>0$.

Furthermore, $f$ and $g$ in (1) can be respectively replaced by a strictly increasing, continuous, and unbounded function $f^{\prime}$ on $\mathbb{R}$ and a strictly increasing and continuous function $g^{\prime}$ on $\mathbb{R}_{+}$ if and only if $f^{\prime}=c f+a$ and $g^{\prime}=c g+b$ for some numbers $a, b$ and a positive $c, g$ in (2) can be replaced by a strictly increasing and continuous function $g^{\prime}$ on $\mathbb{R}_{+}$if and only if $g^{\prime}=g+a$ for some number $a$, and $\theta s$ in (3) and (4) are unique.

The proof of the theorem will be deferred to Appendix B. The final observation for the need of strengthening Axioms A3 and A6 is that constructions of models (b) and (c) heavily depend on model (a), while model (d) can be constructed directly by Axiom B4 without those strengthening.

\section{Conclusion}

A vast amount of research have been devoted to refinements and generalizations of the traditional EU axiomatization, in which the agent's preference relation on a set of random variables is taken as a primitive. However, little is known for similar axiomatization to guarantee the existence of MV utility, although several attempts have been made to characterize MV utility. To my knowledge, only Pollatsek-Tversky's characterization was preferencebased, but they explicitly assume means and variances of random variables in their axiom system.

We developed preference-based axiom systems for the existence of MV utility. We specify preferences for random variables with the same mean-value and show that those preferences obey maximization of EU values of a quadratic utility. The key idea is to apply the constant exchange risk property to the present context. Furthermore, we demonstrated that constancies of attitudes toward risk additively separate effects of mean-values and variances on functional forms of MV utility.

\section{Appendix A}

This appendix proves sufficiency of Theorems 1, 2, and 3. We shall introduce three notations for subsets of $\mathbb{X}$ as follows: $\mathbb{X}^{*}$ is the set of all simple random variables, $\mathbb{X}^{* *}$ is the 
set of all random variables with bounded supports, and $\mathbb{X}^{* * *}$ is the set of all bounded-inpreference random variables. Recall that $\mathbb{Y}_{a}$ is a set of random variables whose mean-values equal $a$. Thus, for example, $\mathbb{X}_{a}^{*}$ is the set of all simple random variables whose mean-values equal $a$.

First, we shall go through a series of lemmas to prove sufficiency of Axioms A1-A7 in Theorem 1 . For $x, y \in \mathbb{R}$, we say that $\lambda$-compound $\langle x ; \lambda ; y\rangle$ is a binary random variable. In what follows, when $\langle x ; \lambda ; y\rangle$ is fair and $x \neq y$, i.e., $\lambda=y /(y-x)>0$, we shall for simplicity delete compound probability $\lambda$ in the notation and denote it by $\langle x, y\rangle$.

Lemma A1. Every random variable in $\mathbb{X}_{0}^{*}$ can be represented by a compound of a finite number of binary fair random variables.

Proof. Take any $X \in \mathbb{X}_{0}^{*}$ for which the support of $X$ is given by $\left\{x_{1}, \ldots, x_{m}, 0,-y_{1}, \ldots,-y_{n}\right\}$ and

$$
\begin{aligned}
p_{X}\left(x_{i}\right) & =\alpha_{i} \quad(i=1, \ldots, m), \\
p_{X}(0) & =\alpha_{0}, \\
p_{X}\left(-y_{j}\right) & =\beta_{j} \quad(j=1, \ldots, n),
\end{aligned}
$$

where $n \geq 1, m \geq 1, x_{i}>0$ for $i=1, \ldots, m, y_{i}>0$ for $i=1, \ldots, n$, and $\sum_{i=1}^{m} \alpha_{i} x_{i}=$ $\sum_{j=1}^{n} \beta_{j} y_{j}$.

For $k=1, \ldots m$, we define $X_{k}$ to be a fair random variable whose support is $\left\{x_{k},-y_{1}, \ldots,-y_{n}\right\}$, so that its density function is given by

$$
\begin{aligned}
p_{X_{k}}\left(x_{k}\right) & =\delta_{k}, \\
p_{X_{k}}\left(-y_{j}\right) & =\gamma_{j} \quad(j=1, \ldots, n),
\end{aligned}
$$

where

$$
\begin{aligned}
\delta_{k} & =\frac{\sum \alpha_{i} x_{i}}{\sum \alpha_{i} x_{i}+x_{k} \sum \beta_{j}}, \\
\gamma_{\ell} & =\frac{x_{k} \beta_{\ell}}{\sum \alpha_{i} x_{i}+x_{k} \sum \beta_{j}} \quad(\ell=1, \ldots, n) .
\end{aligned}
$$

Then $X$ is a $\left(\lambda_{1}, \ldots, \lambda_{m}\right)$-compound of $X_{1}, \ldots, X_{m}$, where, for $k=1, \ldots, m$,

$$
\lambda_{k}=\frac{\alpha_{k}\left(\sum \alpha_{i} x_{i}+x_{k} \sum \beta_{j}\right)}{\sum \alpha_{i} x_{i}} .
$$

Therefore it remains to show that a fair random variable $X$ whose support is given by $\left\{x,-y_{1}, \ldots,-y_{n}\right\}$ is a compound of binary fair gambles $\left\langle x,-y_{1}\right\rangle, \ldots,\left\langle x,-y_{n}\right\rangle$, where $x>0$ and $y_{i}>0$ for $i=1, \ldots, n$. Let $p_{X}(x)=\alpha$ and $p_{X}\left(-y_{i}\right)=\beta_{i}$ for $i=1, \ldots, n$ with 
$\alpha+\sum \beta_{i}=1$. Since $X$ is fair, we have $\alpha x=\sum \beta_{i} y_{i}$. Hence it is easy to see that $X$ is $\left(\lambda_{1}, \ldots, \lambda_{n}\right)$-compound of $\left\langle x,-y_{1}\right\rangle, \ldots,\left\langle x,-y_{n}\right\rangle$, where, for $k=1, \ldots, n$,

$$
\lambda_{k}=\frac{\beta_{k}\left(x+y_{k}\right)}{x} .
$$

This completes the proof of the lemma.

For each $a \in \mathbb{R}$, we say that a real valued function $U_{a}$ on $\mathbb{Y}_{a}$ is linear if, for all $X, Y \in \mathbb{Y}_{a}$ and all $0<\lambda<1$,

$$
U_{a}(\langle X ; \lambda ; Y\rangle)=\lambda U_{a}(X)+(1-\lambda) U_{a}(Y)
$$

where $\mathbb{Y}=\mathbb{X}^{*}$ for Theorem $1, \mathbb{Y}=\mathbb{X}^{* *}$ for Theorem 2 , and $\mathbb{Y}=\mathbb{X}^{* * *}$ for Theorem 3. The existence of $U_{a}$ is proved by the following lemma.

Lemma A2. Axioms A1-A3 hold for ( $\mathbb{Y}, \precsim)$ if and only if, for all $a \in \mathbb{R}$, there is a linear function $U_{a}$ on $\mathbb{Y}_{a}$ such that, for all $X, Y \in \mathbb{Y}_{a}$,

$$
X \precsim Y \Longleftrightarrow U_{a}(X) \leq U_{a}(Y)
$$

Furthermore, $U_{a}$ is unique up to a positive linear transformation.

Proof. Since $\mathbb{Y}_{a}$ is convex, it immediately follows from Lemma 5 in [15].

The following lemma proves that each $U_{a}$ restricted to $\mathbb{X}_{a}^{*}$ can be represented by expected utility form.

Lemma A3. If Axioms A1-A3 hold for $\left(\mathbb{X}^{*}, \precsim\right)$, then, for all $a \in \mathbb{R}$, there is a real valued function $u_{a}$ on $\mathbb{R}$ such that, for all $X, Y \in \mathbb{X}_{a}^{*}$,

$$
X \precsim Y \Longleftrightarrow \sum_{x \in \mathbb{R}} p_{X}(x) u_{a}(x) \leq \sum_{x \in \mathbb{R}} p_{Y}(x) u_{a}(x)
$$

Furthermore, if another real valued function $v_{a}$ on $\mathbb{R}$ represents $\precsim$ as $u_{a}$ does, then there are real numbers $b>0, c$, and $d$ such that, for all $x \in \mathbb{R}$,

$$
v_{a}(x)=b u_{a}(x)+c x+d .
$$

Proof. Assume that axioms A1-A3 hold for $\left(\mathbb{X}^{*}, \precsim\right)$. Let $U_{a}$ be a linear function on $\mathbb{X}_{a}^{*}$ obtained in Lemma A2. By Lemma A1, it suffices to show that there is a real valued function $u_{a}$ on $\mathbb{R}$ such that, for all binary fair random variables $\langle b,-c\rangle$ with $b>0$ and $c>0$,

$$
U_{a}(\langle b,-c\rangle+a)=\frac{c}{b+c} u_{a}(b+a)+\frac{b}{b+c} u_{a}(-c+a) .
$$


Fix $x_{0}>0$. Let $u_{a}\left(x_{0}+a\right)$ be assigned any real number. Then first we define $u_{a}$ on $(-\infty, a)$ to satisfy that, for all $x>0$,

$$
U_{a}\left(\left\langle x_{0},-x\right\rangle+a\right)=\frac{x}{x_{0}+x} u_{a}\left(x_{0}+a\right)+\frac{x_{0}}{x_{0}+x} u_{a}(-x+a) .
$$

Then we define $u_{a}$ on $(a, \infty)$ to satisfy that, for all $x>0$, but $x \neq x_{0}$,

$$
U_{a}\left(\left\langle x,-x_{0}\right\rangle+a\right)=\frac{x_{0}}{x+x_{0}} u_{a}(x+a)+\frac{x}{x+x_{0}} u_{a}\left(-x_{0}+a\right) .
$$

For the time being, we shall fix $x>0$ and $y>0$. Let $X$ and $Y$ be compound random variables defined by

$$
\begin{aligned}
& X=\left\langle\left\langle x_{0},-x_{0}\right\rangle+a ; \frac{2 x y}{K} ;\langle x,-y\rangle+a\right\rangle, \\
& Y=\left\langle\left\langle x,-x_{0}\right\rangle+a ; \frac{y\left(x+x_{0}\right)}{K} ;\left\langle x_{0},-y\right\rangle+a\right\rangle,
\end{aligned}
$$

where $K=x\left(x_{0}+y\right)+y\left(x+x_{0}\right)$. Then $X, Y \in \mathbb{X}_{a}^{*}$, so that

$$
\begin{aligned}
& U_{a}(X)=\frac{2 x y}{K} U_{a}\left(\left\langle x_{0},-x_{0}\right\rangle+a\right)+\frac{x_{0}(x+y)}{K} U_{a}(\langle x,-y\rangle+a), \\
& U_{a}(Y)=\frac{y\left(x+x_{0}\right)}{K} U_{a}\left(\left\langle x,-x_{0}\right\rangle+a\right)+\frac{x\left(x_{0}+y\right)}{K} U_{a}\left(\left\langle x_{0},-y\right\rangle+a\right) .
\end{aligned}
$$

Since $p_{X}(x)=p_{Y}(x)$ for all $x \in \mathbb{R}$, we have $U_{a}(X)=U_{a}(Y)$, so that

$$
\begin{aligned}
& 2 x y U_{a}\left(\left\langle x_{0},-x_{0}\right\rangle+a\right)+x_{0}(x+y) U_{a}(\langle x,-y\rangle+a) \\
& =y\left(x+x_{0}\right) U_{a}\left(\left\langle x,-x_{0}\right\rangle+a\right)+x\left(x_{0}+y\right) U_{a}\left(\left\langle x_{0},-y\right\rangle+a\right) .
\end{aligned}
$$

Substituting $u_{a}$-expressions of $U_{a}\left(\left\langle x_{0},-y\right\rangle+a\right)$ and $U_{a}\left(\left\langle x,-x_{0}\right\rangle+a\right)$ in the preceding paragraph for this, its rearrangement gives

$$
U_{a}(\langle x,-y\rangle+a)=\frac{y}{x+y} u_{a}(x+a)+\frac{x}{x+y} u_{a}(-y+a) .
$$

Since $x>0$ and $y>0$ are arbitrary, this completes the representation part of the lemma.

Uniqueness proof goes as follows. Suppose that $V_{a}(X)=\sum_{x \in \mathbb{R}} p_{X}(x) v_{a}(x)$ represent $\precsim$. Then

$$
V_{a}\left(\left\langle x_{0},-x\right\rangle+a\right)=\frac{x}{x_{0}+x} v_{a}\left(x_{0}+a\right)+\frac{x_{0}}{x_{0}+x} v_{a}(-x+a) .
$$

By Lemma A2, $V_{a}(X)=\alpha U_{a}(X)+\beta$ for some $\alpha>0$ and $\beta$. Then 


$$
\begin{aligned}
v_{a}(-x+a) & =\frac{x_{0}+x}{x_{0}}\left[V_{a}\left(\left\langle x_{0},-x\right\rangle+a\right)-\frac{x}{x_{0}+x} v_{a}\left(x_{0}+a\right)\right] \\
& =\frac{x_{0}+x}{x_{0}}\left[\alpha U_{a}\left(\left\langle x_{0},-x\right\rangle+a\right)+\beta-\frac{x}{x_{0}+x} v_{a}\left(x_{0}+a\right)\right] \\
& =\frac{x_{0}+x}{x_{0}}\left[\alpha\left(\frac{x}{x_{0}+x} u_{a}\left(x_{0}+a\right)+\frac{x_{0}}{x_{0}+x} u_{a}(-x+a)\right)+\beta-\frac{x}{x_{0}+x} v_{a}\left(x_{0}+a\right)\right] \\
& =\frac{\alpha u_{a}\left(x_{0}+a\right)-v_{a}\left(x_{0}+a\right)+\beta}{x_{0}} x+\alpha u_{a}(-x+a)+\beta
\end{aligned}
$$

Hence the uniqueness obtains.

In fact, $u_{a}$ in Lemma A3 turns out to be quadratic as shown by the following lemma.

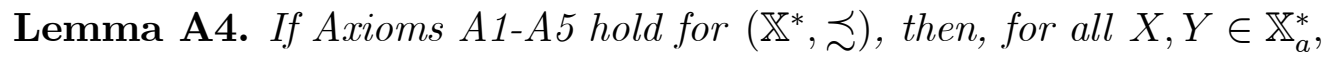

$$
X \precsim Y \Longleftrightarrow-\sigma_{X}^{2} \leq-\sigma_{Y}^{2}
$$

Proof. Let $u_{a}$ be a real valued function on $\mathbb{R}$ obtained in Lemma A3. It suffices to show that $u_{a}(x+a)=-x^{2}$. First we show that $u_{a}$ can be scaled as a symmetric function around $a$. With no loss of generality, we assume that $u_{a}(a)=0$. Fix $x_{0}>0$. Assume $u_{a}\left(x_{0}+a\right) \neq u_{a}\left(-x_{0}+a\right)$. Then letting $\alpha=\frac{1}{2 x_{0}}\left(u_{a}\left(-x_{0}+a\right)-u_{a}\left(x_{0}+a\right)\right)$, we get

$$
u_{a}\left(x_{0}+a\right)+\alpha x_{0}=u_{a}\left(-x_{0}+a\right)-\alpha x_{0} .
$$

Thus because of the uniqueness of $u_{a}$ in Lemma A3, this implies that we can assume $u_{a}\left(x_{0}+a\right)=u_{a}\left(-x_{0}+a\right)$, so that symmetry of $u_{a}$ around $a$ follows. To see this, we note, by Axiom A4, that $\left\langle x,-x_{0}\right\rangle+a \sim\left\langle x_{0},-x\right\rangle+a$. Thus, by Lemma A3, we obtain that

$$
\frac{x_{0}}{x+x_{0}} u_{a}(x+a)+\frac{x}{x+x_{0}} u_{a}\left(-x_{0}+a\right)=\frac{x}{x+x_{0}} u_{a}\left(x_{0}+a\right)+\frac{x_{0}}{x+x_{0}} u_{a}(-x+a),
$$

which gives

$$
u_{a}(x+a)-u_{a}(-x+a)=\frac{u_{a}\left(x_{0}+a\right)-u_{a}\left(-x_{0}+a\right)}{x_{0}} x
$$

Hence $u_{a}(x+a)=u_{a}(-x+a)$ for all $x$.

In what follows, we shall prove that $u_{a}(x+a)=-b x^{2}$ for some $b>0$. It follows from Axiom A5 that, for $X \in \mathbb{X}_{a}^{*}$ and $\delta>0$,

$$
\left\langle X-\delta ; \frac{1}{2} ; a+\delta\right\rangle \sim\left\langle a-\delta ; \frac{1}{2} ; X+\delta\right\rangle
$$

since $\mu_{X}=a$. Then 


$$
\sum p_{X}(x)\left(u_{a}(x+\delta)-u_{a}(x-\delta)\right)=u_{a}(a+\delta)-u_{a}(a-\delta) .
$$

By symmetry of $u_{a}$ around $a, u_{a}(a+\delta)=u_{a}(a-\delta)$, so that

$$
\sum p_{X}(x)\left(u_{a}(x+\delta)-u_{a}(x-\delta)\right)=0
$$

Then, when $X=\left\langle x,-x_{0}\right\rangle+a$, we get

$$
\frac{x_{0}}{x+x_{0}}\left[u_{a}(x+a+\delta)-u_{a}(x+a-\delta)\right]+\frac{x}{x+x_{0}}\left[u_{a}\left(-x_{0}+a+\delta\right)-u_{a}\left(-x_{0}+a-\delta\right)\right]=0,
$$

SO

$$
u_{a}(x+a+\delta)-u_{a}(x+a-\delta)=-\frac{x}{x_{0}}\left[u_{a}\left(-x_{0}+a+\delta\right)-u_{a}\left(-x_{0}+a-\delta\right)\right] .
$$

Let $\phi(\delta)=-\frac{1}{x_{0}}\left[u_{a}\left(-x_{0}+a+\delta\right)-u_{a}\left(-x_{0}+a-\delta\right)\right]$. Hence

$$
u_{a}(x+a+\delta)-u_{a}(x+a-\delta)=\phi(\delta) x
$$

for some $\phi(\delta) \neq 0$.

Substituting $(2 k-1) \delta$ for $x$ in the last equation of the previous paragraph, we obtain that, for $k=1,2, \ldots$,

$$
u_{a}(2 k \delta+a)-u_{a}(2(k-1) \delta+a)=(2 k-1) \delta \phi(\delta)
$$

so that summation over $k$ from 1 through $m$ gives

$$
u_{a}(2 m \delta+a)=m^{2} \delta \phi(\delta) .
$$

If $\phi(\delta)=-\alpha \delta$ for some $\alpha>0$, then

$$
u_{a}(2 m \delta+a)=-\frac{\alpha}{4}(2 m \delta)^{2} .
$$

Since $\delta>0$ is arbitrary and $u_{a}$ is symmetric, we obtain the desired result, i.e., $u_{a}(x+a)=$ $-b x^{2}$ for some $b>0$.

In what follows, we shall show that $\phi(\delta)=-\alpha \delta$ for some $\alpha>0$. Substituting $\frac{\delta}{n}$ for $\delta$ in (1), we get

$$
u_{a}\left(\frac{2 m \delta}{n}+a\right)=\frac{m^{2} \delta}{n} \phi\left(\frac{\delta}{n}\right)
$$

For $m=n \ell$ in $(2)$, we get

$$
u_{a}(2 \ell \delta+a)=n \ell^{2} \delta \phi\left(\frac{\delta}{n}\right) .
$$

On the other hand, (1) for $m=\ell$ is $u_{a}(2 \ell \delta+a)=\ell^{2} \delta \phi(\delta)$. Hence, 


$$
\phi\left(\frac{\delta}{n}\right)=\frac{1}{n} \phi(\delta) .
$$

Substituting $\frac{k}{n} \delta$ for $\delta$ in (1), we get

$$
u_{a}\left(\frac{2 k m \delta}{n}+a\right)=\frac{m^{2} k \delta}{n} \phi\left(\frac{k}{n} \delta\right) .
$$

Substituting $\mathrm{km}$ for $m$ in (2), we get

$$
\begin{aligned}
u_{a}\left(\frac{2 k m \delta}{n}+a\right) & =\frac{k^{2} m^{2} \delta}{n} \phi\left(\frac{\delta}{n}\right) \\
& =\frac{k^{2} m^{2} \delta}{n^{2}} \phi(\delta) .
\end{aligned}
$$

Hence,

$$
\phi\left(\frac{k}{n} \delta\right)=\frac{k}{n} \phi(\delta) .
$$

If $\phi(x)$ is strictly decreasing in $x>0$, we must have that $\phi(\lambda x)=\lambda \phi(x)$ for all $\lambda>0$. Hence $\phi(x)=-\alpha x$ for some $\alpha>0$.

It remains to show that $\phi(x)$ is strictly decreasing in $x>0$. Let $0<\delta<\delta^{\prime}, R=$ $\left\langle\delta ; \frac{1}{2} ;-\delta^{\prime}\right\rangle$, and $S=\left\langle-\delta ; \frac{1}{2} ; \delta^{\prime}\right\rangle$. Then $\mu_{R+a}<\mu_{S+a}$. Thus by Axiom A5,

$$
\left\langle R+a-x_{0} ; \frac{1}{2} ; S+a+x_{0}\right\rangle \prec\left\langle S+a-x_{0} ; \frac{1}{2} ; R+a+x_{0}\right\rangle,
$$

where those $\frac{1}{2}$-compound random variables belong to $\mathbb{X}_{0}^{*}$. Thus we have

$$
\begin{aligned}
& u_{a}\left(-x_{0}+\delta+a\right)+u_{a}\left(-x_{0}-\delta^{\prime}+a\right)+u_{a}\left(x_{0}-\delta+a\right)+u_{a}\left(x_{0}+\delta^{\prime}+a\right) \\
& <u_{a}\left(-x_{0}-\delta+a\right)+u_{a}\left(-x_{0}+\delta^{\prime}+a\right)+u_{a}\left(x_{0}+\delta+a\right)+u_{a}\left(x_{0}-\delta^{\prime}+a\right),
\end{aligned}
$$

which by symmetry of $u_{a}$ around $a$ implies

$$
u_{a}\left(-x_{0}+\delta+a\right)+u_{a}\left(-x_{0}-\delta^{\prime}+a\right)<u_{a}\left(-x_{0}-\delta+a\right)+u_{a}\left(-x_{0}+\delta^{\prime}+a\right)
$$

so that

$$
\begin{aligned}
\phi\left(\delta^{\prime}\right) & =\frac{1}{x_{0}}\left(u_{a}\left(-x_{0}-\delta^{\prime}+a\right)-u_{a}\left(-x_{0}+\delta^{\prime}+a\right)\right) \\
& <\phi(\delta)=\frac{1}{x_{0}}\left(u_{a}\left(-x_{0}-\delta+a\right)-u_{a}\left(-x_{0}+\delta+a\right)\right)
\end{aligned}
$$

Hence $\phi$ is strictly decreasing. 
Sufficiency Proof of Theorem 1. Suppose that $\mathbb{Y}=\mathbb{X}^{*}$ and Axioms A1-A7 hold for ( $\mathbb{Y}, \precsim)$. Then it follows from Lemma A4 that, for all $X, Y \in \mathbb{Y}$,

$$
\mu_{X}=\mu_{Y} \text { and } \sigma_{X}^{2}=\sigma_{Y}^{2} \Longrightarrow X \sim Y \text {. }
$$

For all $X \in \mathbb{Y}$, let $c_{X}$ denote the certainty equivalent of $X$, i.e., $X \sim c_{X}$, whose unique existence is assured by Axioms $\mathrm{A} 6$ and A7. Therefore, by Axiom A7, for all $X, Y \in \mathbb{Y}$, $X \precsim Y \Longleftrightarrow c_{X} \leq c_{Y}$. Thus we define a real valued function $U$ on $\mathbb{R} \times \mathbb{R}_{+}$as follows: for all $X \in \mathbb{Y}$,

$$
U\left(\mu_{X}, \sigma_{X}^{2}\right)=c_{X}
$$

Hence, for all $X, Y \in \mathbb{Y}$,

$$
X \precsim Y \Longleftrightarrow U\left(\mu_{X}, \sigma_{X}^{2}\right) \leq U\left(\mu_{Y}, \sigma_{Y}^{2}\right) .
$$

By Axiom A7 and Lemma A4, it is easy to see that $U(\alpha, \beta)$ is strictly increasing in $\alpha$ and strictly decreasing in $\beta$.

Sufficiency Proofs of Theorems 2 and 3. In what follows, we assume that Axioms A1-A7 hold for ( $\mathbb{Y}, \precsim)$. By Lemmas A2 and A4, there exist linear functions $U_{a}$ on $\mathbb{Y}_{a}$ for all $a \in \mathbb{R}$ such that

(1) for all $X, Y \in \mathbb{Y}_{a}, X \precsim Y \Longleftrightarrow U_{a}(X) \leq U_{a}(Y)$;

(2) for all $X \in \mathbb{X}_{a}^{*}, U_{a}(X)=-\sigma_{X}^{2}$,

where $\mathbb{Y}=\mathbb{X}^{* *}$ for Theorem 2 and $\mathbb{Y}=\mathbb{X}^{* * *}$ for Theorem 3. Note that, for any $X \in \mathbb{Y}_{0}$, $\left\langle X ; \frac{1}{2} ;-X\right\rangle$ is symmetric and belongs to $\mathbb{Y}_{0}$. Hence, by linearity of $U_{0}$, we have

$$
U_{0}\left(\left\langle X ; \frac{1}{2} ;-X\right\rangle\right)=\frac{1}{2}\left(U_{0}(X)+U_{0}(-X)\right) .
$$

By Axiom A4, $U_{0}(X)=U_{0}(-X)$, so that $U_{0}\left(\left\langle X ; \frac{1}{2} ;-X\right\rangle\right)=U_{0}(X)$. Since, by Axioms A8 and A9, dominance with respect to mean-preserving spreads and truncation dominance respectively hold at any wealth level, it suffices to prove that $U_{0}(X)=-\sigma_{X}^{2}>-\infty$ for all symmetric $X$ which belong to $\mathbb{X}_{0}^{* *} \backslash \mathbb{X}_{0}^{*}$ for sufficiency proof of Theorem 2 and $\mathbb{X}_{0}^{* * *} \backslash \mathbb{X}_{0}^{* *}$ for sufficiency proof of Theorem 3.

Assume that Axiom A8 holds. Consider a symmetric $X \in \mathbb{X}_{0}^{* *} \backslash \mathbb{X}_{0}^{*}$. We show that there exist two sequences of simple symmetric random variables, $\left\{Y_{n}\right\} \subset \mathbb{X}_{0}^{*}$ and $\left\{Z_{n}\right\} \subset \mathbb{X}_{0}^{*}$, such that $Y_{n} \precsim X \precsim Z_{n}$ for all $n \in N, \sigma_{Y_{n}}^{2} \geq \sigma_{Y_{n+1}}^{2} \geq \sigma_{Z_{n+1}}^{2} \geq \sigma_{Z_{n}}^{2}$ for all $n \in N$, and $\lim _{n \rightarrow \infty} \sigma_{Y_{n}}^{2}=\lim _{n \rightarrow \infty} \sigma_{Z_{n}}^{2}=\sigma_{X}^{2}$. With no loss of generality, we assume that $p_{X}([-1,1])=1$. Let $x_{n, k}=\sqrt{\frac{k}{2^{n}}}$ for $n \in N$ and $k=0,1, \ldots, 2^{n}$. For $n \in N$, let $Y_{n}$ and $Z_{n}$ be simple random variables whose density functions are given as follows: for $k=1, \ldots, 2^{n}$ and $\ell=1, \ldots, 2^{n}-1$, 


$$
\begin{aligned}
p_{Y_{n}}\left(x_{n, k}\right) & =p_{X}\left(\left(x_{n, k-1}, x_{n, k}\right]\right), \\
p_{Y_{n}}\left(-x_{n, k}\right) & =p_{X}\left(\left[-x_{n, k},-x_{n, k-1}\right)\right), \\
p_{Y_{n}}(0) & =p_{X}(0), \\
p_{Z_{n}}\left(x_{n, \ell}\right) & =p_{X}\left(\left(x_{n, \ell}, x_{n, \ell+1}\right]\right), \\
p_{Z_{n}}\left(-x_{n, \ell}\right) & =p_{X}\left(\left[-x_{n, \ell+1},-x_{n, \ell}\right)\right), \\
p_{Z_{n}}(0) & =p_{X}\left(\left[-x_{n, 1}, x_{n, 1}\right]\right) .
\end{aligned}
$$

Since $X$ is symmetric, so are $Y_{n}$ and $Z_{n}$ for all $n \in N$. Thus $\left\{Y_{n}\right\} \subset \mathbb{X}_{0}^{*}$ and $\left\{Z_{n}\right\} \subset \mathbb{X}_{0}^{*}$. Note that, for all $n \in N, Y_{n}$ is a mean-preserving spread of $Y_{n+1}$ and $Z_{n+1}$ is a mean-preserving spread of $Z_{n}$. Also, note that, for all $n \in N, Y_{n}$ is a mean-preserving spread of $X$ and $X$ is a mean-preserving spread of $Z_{n}$. Hence, by Axiom A8, for all $n \in N$,

$$
Y_{n} \precsim Y_{n+1} \precsim X \precsim Z_{n+1} \precsim Z_{n}
$$

Since $Y_{n}, Z_{n} \in \mathbb{X}_{0}^{*}$, it follows from Theorem 1 that $\inf _{n} \sigma_{Y_{n}}^{2} \geq \sup _{n} \sigma_{Z_{n}}^{2}$. Therefore, for all $n \in N$,

$$
\begin{aligned}
& -\sigma_{Y_{n}}^{2}=-\sum_{k=1}^{2^{n}} \frac{k}{2^{n}}\left\{p_{Y_{n}}\left(\left(x_{n, k-1}, x_{n, k}\right]\right)+p_{Y_{n}}\left(\left[-x_{n, k},-x_{n, k-1}\right)\right)\right\} \\
& \leq U_{0}(X) \leq-\sigma_{Z_{n}}^{2}=-\sum_{k=1}^{2^{n}-1} \frac{k}{2^{n}}\left\{p_{Z_{n}}\left(\left(x_{n, k}, x_{n, k+1}\right]\right)+p_{Z_{n}}\left(\left[-x_{n, k+1},-x_{n, k}\right)\right)\right\}
\end{aligned}
$$

Since the difference between the two sums above is not greater than $\frac{1}{2^{n}}$, which vanishes as $n$ gets large, it follows from the definition of integration that

$$
U_{0}(X)=\int_{-1}^{1} x^{2} d p_{X}(x)=-\sigma_{X}^{2} .
$$

This completes the sufficiency proof of Theorem 2.

To prove sufficiency of Theorem 3, we assume that Axioms 8 and 9 hold. Consider a symmetric $X \in \mathbb{X}_{0}^{* * *} \backslash \mathbb{X}_{0}^{* *}$. Since $X$ is bounded in preference, there exists an $a \geq 0$ such that $\langle a,-a\rangle \precsim X$. By Axiom A8, $X \precsim X_{b, \infty}$ for all $b \geq 0$. Since $X_{b, \infty} \in \mathbb{X}_{0}^{* *}$, we obtain that

$$
U_{0}(\langle a,-a\rangle) \leq U_{0}(X) \leq \inf _{b} U_{0}\left(X_{b, \infty}\right)=-\int_{-\infty}^{\infty} x^{2} d p_{X}(x) .
$$

This implies that variance of $X$ is bounded. Assume that

$$
U_{0}(X)<-\int_{-\infty}^{\infty} x^{2} d p_{X}(x) .
$$


Then there exists a $c>0$ such that $X \prec\langle c,-c\rangle \prec X_{b, \infty}$ for all $b \geq 0$. By Axiom A9, $X_{d, \infty} \prec\langle c,-c\rangle$ for some $d \geq 0$. This is a contradiction. Hence, we must have $U_{0}(X)=$ $-\int_{-\infty}^{\infty} x^{2} d p_{X}(x)$. This completes the sufficiency proof of Theorem 3 .

\section{Appendix B}

Recall that $\mathbb{X}^{*}$ is the set of all simple random variables. We prove Theorem 4 . In what follows we shall assume that Axioms A1, A2, A3*, A4, A5, A6*, and A7 hold for ( $\left.\mathbb{X}^{*}, \precsim\right)$.

(1) Necessity of Axiom B1 easily obtains. Thus we shall prove its sufficiency. For notational abuse, by $\precsim$, we shall denote the binary relation on $\mathbb{R} \times \mathbb{R}_{+}$induced by $\precsim$ on $\mathbb{X}^{*}$ as follows: for all $(\alpha, a),(\beta, b) \in \mathbb{R} \times \mathbb{R}_{+}$,

$$
\begin{aligned}
(\alpha, a) \precsim(\beta, b) \Longleftrightarrow & X \precsim Y \text { for some } X, Y \in \mathbb{X}^{*} \text { with } \\
& (\alpha, a)=\left(\mu_{X}, \sigma_{X}^{2}\right) \text { and }(\beta, b)=\left(\mu_{Y}, \sigma_{Y}^{2}\right) .
\end{aligned}
$$

Then it suffices to show that there exist a strictly increasing, continuous, and unbounded function $f$ on $\mathbb{R}$, and a strictly increasing and continuous function $g$ on $\mathbb{R}_{+}$such that, for all $(\alpha, a),(\beta, b) \in \mathbb{R} \times \mathbb{R}_{+}$,

$$
(\alpha, a) \precsim(\beta, b) \Longleftrightarrow f(\alpha)-g(a) \leq f(\beta)-g(b) .
$$

It follows from [11] that sufficient axioms for the existence of $f$ and $g$ are given by the following six conditions, C1-C6, which are understood as applying to all $\alpha, \beta, \gamma \in \mathbb{R}$ and $a, b, c \in \mathbb{R}_{+}$.

C1. (order). $\precsim$ is a weak order.

C2. (independence). If $(\alpha, a) \precsim(\beta, a)$, then $(\alpha, b) \precsim(\beta, b)$; if $(\alpha, a) \precsim(\alpha, b)$, then $(\beta, a) \precsim(\beta, b)$.

C3. (Thomsen condition). If $(\alpha, c) \sim(\gamma, b)$ and $(\gamma, a) \sim(\beta, c)$, then $(\alpha, a) \sim(\beta, b)$.

C4. (restricted solvability). If $(\beta, b) \precsim(\alpha, a) \precsim(\gamma, b)$, then $(\delta, b) \sim(\alpha, a)$ for some $\delta \in \mathbb{R}$; if $(\beta, b) \precsim(\alpha, a) \precsim(\beta, c)$, then $(\beta, d) \sim(\alpha, a)$ for some $d \in \mathbb{R}_{+}$.

C5. (Archimedeanity). Every bounded standard sequence is finite, where $\left\{\alpha_{i} \in \mathbb{R}: i \in K\right\}$ (respectively, $\left\{a_{i} \in \mathbb{R}_{+}: i \in K\right\}$ ) is said to be a standard sequence if there exist $d, e \in \mathbb{R}$ (resp., $\delta, \epsilon \in \mathbb{R}_{+}$) such that $d \neq e\left(\right.$ resp., $\delta \neq \epsilon$ ) and for all $i, i+1 \in K,\left(\alpha_{i}, d\right) \sim\left(\alpha_{i+1}, e\right)$ (resp., $\left.\left(\delta, a_{i}\right) \sim\left(\epsilon, a_{i+1}\right)\right)$ for a set $K$ of consecutive integers.

C6. (Essentiality). $\neg((\delta, d) \sim(\epsilon, d))$ for some $\delta, \epsilon \in \mathbb{R}$ and some $d \in \mathbb{R}_{+} ; \neg((\delta, d) \sim(\delta, e))$ for some $\delta \in \mathbb{R}$ and some $d, e \in \mathbb{R}_{+}$. 
We prove that Axioms A1, A2, A3*, A4, A5, A6*, A7, and B1 imply C1-C6. C1 follows from Axiom A1. The first part of $\mathrm{C} 2$ follows from Axiom A7. The second part of $\mathrm{C} 2$ follows from Lemma A4, which follows from Axioms A1-A5, in Appendix A. To prove C3, suppose that $(\alpha, c) \sim(\gamma, b)$ and $(\gamma, a) \sim(\beta, c)$. Then take any $X, Y, Z \in \mathbb{X}^{*}$ such that $\gamma=\mu_{X}=\mu_{X}=\mu_{Z}, b=\sigma_{X}^{2}, a=\sigma_{Y}^{2}$, and $c=\sigma_{Z}^{2}$. Thus $X, Y, Z \in X_{\gamma}^{*}$ and

$$
\begin{aligned}
& (\alpha, c) \sim(\gamma, b) \Longrightarrow\left(\mu_{Z}+\left(\alpha-\mu_{Z}\right), \sigma_{Z}^{2}\right) \sim\left(\mu_{X}, \sigma_{X}^{2}\right), \\
& (\gamma, a) \sim(\beta, c) \Longrightarrow\left(\mu_{Y}, \sigma_{Y}^{2}\right) \sim\left(\mu_{Z}+\left(\beta-\mu_{Z}\right), \sigma_{Z}^{2}\right),
\end{aligned}
$$

which imply $X \sim Z+\alpha-\gamma$ and $Y \sim Z+\beta-\gamma$. By Axiom B1, $X+\beta-\gamma \sim Y+\alpha-\gamma$, so that $\left(\beta, \sigma_{X}^{2}\right) \sim\left(\alpha, \sigma_{Y}^{2}\right)$. Hence, $(\beta, b) \sim(\alpha, a)$, so that Thomsen condition obtains.

The first part of $\mathrm{C} 4$ follows from Axioms $\mathrm{A} 6^{*}$ and A7. To see this, take any $X \in \mathbb{X}_{0}^{*}$, $Z \in \mathbb{X}^{*}$, and $a \in \mathbb{R}$. Then by Axiom A6 $6^{*}, Z \sim X+a+b$ for some $b \in \mathbb{R}$. Hence, $\left(a+b^{\prime}, \sigma_{X}^{2}\right) \precsim\left(\mu_{Z}, \sigma_{Z}^{2}\right) \precsim\left(a+b^{\prime \prime}, \sigma_{X}^{2}\right)$ for some $b^{\prime} \leq b \leq b^{\prime \prime}$, and $\left(\mu_{Z}, \sigma_{Z}^{2}\right) \sim\left(a+b, \sigma_{X}^{2}\right)$, so that the desired result obtains. The second part of C4 follows from Axioms A3*. To check this, take any $X, Y \in \mathbb{X}_{0}^{*}, Z \in \mathbb{X}^{*}$, and $a \in \mathbb{R}$, which satisfy that $X+a \precsim Z \precsim Y+a$. Then by Axiom A3*, $Z \sim\langle X+a ; \alpha ; Y+a\rangle$ for some $0<\alpha<1$. Hence $\left(a, \sigma_{X}^{2}\right) \precsim\left(\mu_{Z}, \sigma_{Z}^{2}\right) \precsim\left(a, \sigma_{Y}^{2}\right)$ and $\left(\mu_{Z}, \sigma_{Z}^{2}\right) \sim\left(a, \alpha \sigma_{X}^{2}+(1-\alpha) \sigma_{Y}^{2}\right)$, so that the desired result obtains.

For C5, we show first that any bounded standard sequence on $\mathbb{R}$ is finite. Take any $X, Y \in \mathbb{X}_{0}^{*}$ with $\sigma_{X}^{2} \neq \sigma_{Y}^{2}$, and $a \in \mathbb{R}$. Assume that $\sigma_{X}^{2}<\sigma_{Y}^{2}$. When $\sigma_{Y}^{2}<\sigma_{X}^{2}$, the proof is similar. Then by Axiom A6* $a+X \sim \alpha_{1}+a+Y$ for some $\alpha_{1}>0$. Thus we define a sequence $\alpha_{2}, \alpha_{3}, \ldots$ recursively by the indifference relations $\alpha_{k}+a+X \sim \alpha_{k+1}+a+Y$ for $k \in N$, so that $\alpha_{1}<\alpha_{2}<\cdots$, an increasing infinite sequence. Assume that $\left\{\alpha_{i}\right\}$ is bounded. Then $\sup _{k} \alpha_{k}$ exists. Let $\alpha^{*}=\sup _{k} \alpha_{k}$, so $\alpha_{k}<\alpha^{*}$ for all $k$. Note that $\alpha_{k}+a+X \prec \alpha^{*}+a+Y$. However, By Axiom A6*,$a+\beta+X \sim \alpha^{*}+a+Y$ for some $\beta<\alpha^{*}$. This is a contradiction, so that $\left\{\alpha_{i}\right\}$ must be unbounded. Hence, any bounded standard sequence on $\mathbb{R}$ must be finite.

Next we show that any bounded standard sequence on $\mathbb{R}_{+}$is finite. Take any $a, b \in \mathbb{R}$ with $a \neq b$, and $X, Y \in \mathbb{X}_{0}^{*}$ with $\sigma_{X}^{2}<\sigma_{Y}^{2}$ and $Y+b \precsim X+a \precsim b$. Then, by Axiom $\mathrm{A} 3^{*}, a+X \sim\left\langle Y ; \alpha_{1} ; 0\right\rangle+b$ for some $0 \leq \alpha_{1} \leq 1$. Thus we define a sequence, $\alpha_{2}, \alpha_{3}, \ldots$, recursively by the indifference relations $\left\langle Y ; \alpha_{k} ; 0\right\rangle+a \sim\left\langle Y ; \alpha_{k+1} ; 0\right\rangle+b$ for $k=1,2, \ldots$ as long as $b+Y \precsim\left\langle Y ; \alpha_{k} ; 0\right\rangle+a \precsim b$ (i.e., $\left.U\left(b, \sigma_{Y}^{2}\right) \leq U\left(a, \alpha_{k} \sigma_{Y}^{2}\right) \leq U(b, 0)\right)$. Let $K$ be the set of $k \mathrm{~s}$ thus defined. It may be finite or infinite. For all $k \in K$, let $X_{k}=\left\langle Y ; \alpha_{k} ; 0\right\rangle$. Then $\mu_{X_{k}}=0$ and $\sigma_{X_{k}}^{2}=\alpha_{k} \sigma_{Y}^{2}$. It follows from the indifference relations which define the sequence that $U\left(a, \sigma_{X}^{2}\right)=U\left(b, \sigma_{X_{1}}^{2}\right)$ and $U\left(a, \sigma_{X_{k}}^{2}\right)=U\left(b, \sigma_{X_{k+1}}^{2}\right)$ for $k \in K$, so that $U\left(a, \sigma_{X}^{2}\right)=$ $U\left(b, \alpha_{1} \sigma_{Y}^{2}\right)$ and $U\left(a, \alpha_{k} \sigma_{Y}^{2}\right)=U\left(b, \alpha_{k+1} \sigma_{Y}^{2}\right)$ for $k \in K$. Therefore, $\left\{\alpha_{k} \sigma_{Y}^{2}: k \in K\right\}$ is a bounded standard sequence on $\mathbb{R}_{+}$. We note that any bounded standard sequence on $\mathbb{R}_{+}$ can be constructed in this way.

Suppose that $K$ is infinite. Assume that $a<b$. When $b<a$, the proof is similar. Then $\sigma_{X}^{2}<\sigma_{X_{k}}^{2}<\sigma_{X_{k+1}}^{2}$ for $k \geq 1$, so $\left\{\alpha_{k}: k \in K\right\}$ is a bounded increasing sequence. Let $\alpha_{\infty}=\sup _{k} \alpha_{k}$ and $X_{\infty}=\left\langle Y ; \alpha_{\infty} ; 0\right\rangle$, so $\mu_{X_{\infty}}=0$ and $\sigma_{X_{\infty}}^{2}=\alpha_{\infty} \sigma_{Y}^{2}$. Since $X_{\infty}+$ $a \prec X_{\infty}+b \prec X+a$, Axiom A3* implies that $X_{\infty}+b \sim\left\langle X+a ; \beta ; X_{\infty}+a\right\rangle$ for some 
$0<\beta<1$. Let $Z=\left\langle X ; \beta ; X_{\infty}\right\rangle$. Then $\mu_{Z}=0$ and $\sigma_{Z}^{2}=\beta \sigma_{X}^{2}+(1-\beta) \sigma_{X_{\infty}}^{2}$, so that $\sigma_{Z}^{2}<\sigma_{X_{n}}^{2}<\sigma_{X_{n+1}}^{2}<\sigma_{X_{\infty}}^{2}$ for some $n \in K$. However, $X_{\infty}+b \prec X_{n+1}+b \sim X_{n}+a \prec Z+a$, so that $X_{\infty}+b \prec Z+a$. This contradicts $X_{\infty}+b \sim Z+a$. Hence $K$ is finite.

C6 obtains as follows. By Axiom A7, $a+X \prec b+X$ for some $a, b \in \mathbb{R}$ and some $X \in \mathbb{X}_{0}^{*}$. Thus $\neg\left(\left(a, \sigma_{X}^{2}\right) \sim\left(b, \sigma_{X}^{2}\right)\right)$. By Axiom A5, $\left\langle X-a ; \frac{1}{2} ; Y+a\right\rangle \prec\left\langle Y-a ; \frac{1}{2} ; X+a\right\rangle$ whenever $\mu_{X}<\mu_{Y}$. Let $X^{\prime}=\left\langle X-a ; \frac{1}{2} ; Y+a\right\rangle$ and $Y^{\prime}=\left\langle Y-a ; \frac{1}{2} ; X+a\right\rangle$. Then $\mu_{X^{\prime}}=\mu_{Y^{\prime}}$ and $\sigma_{X^{\prime}}^{2}>\sigma_{Y}^{2}$. Thus $\neg\left(\left(b, \sigma_{X}^{2}\right) \sim\left(b, \sigma_{Y}^{2}\right)\right)$, where $b=\mu_{X^{\prime}}$. This completes the proofs of C1-C6.

Since C1-C6 hold true, we have $U\left(\mu_{X}, \sigma_{X}^{2}\right)=f\left(\mu_{X}\right)-g\left(\sigma_{X}^{2}\right)$. By Axiom A7, $a<b \Longrightarrow$ $X+a \prec X+b$ for $X \in \mathbb{X}_{0}^{*}$, so that $f(a)<f(b)$. Thus $f$ is strictly increasing function $f$ on $\mathbb{R}$. By Lemma A4, $g$ is strictly increasing on $\mathbb{R}_{+}$.

Next we show continuity of $f$ and $g$. We say that $f$ has a gap at $a \in \mathbb{R}$ if $\lim _{i \rightarrow \infty} f\left(a_{i}\right)<$ $\lim _{i \rightarrow \infty} f\left(b_{i}\right)$ whenever $a_{k}<a_{k+1}<a<b_{k+1}<b_{k}$ for all $k$ and $\lim _{i \rightarrow \infty} a_{i}=\lim _{i \rightarrow \infty} b_{i}=a$. When $f$ has no gap at $a \in \mathbb{R}, f$ is continuous at $a$. A gap of $g$ at $a \in \mathbb{R}_{+}$is similarly defined. Since there are at most countable number of points in $\mathbb{R}$ at which $f$ and $g$ have gaps, there exist points in $\mathbb{R}$ (respectively, $\mathbb{R}_{+}$) at which $f$ (respectively, $g$ ) is continuous.

Suppose that $f$ has a gap at $a$. Let $\left\{a_{i}\right\}$ (respectively, $\left\{b_{i}\right\}$ ) be a strictly increasing (respectively, decreasing) sequence which converges to $a$ from below (respectively, above). Assume that $\lim _{i \rightarrow \infty} f\left(a_{i}\right)<f(a)$. We derive a contradiction. When $\lim _{i \rightarrow \infty} f\left(b_{i}\right)>f(a)$, the proof is similar. Let $b \in \mathbb{R}_{+}$be a point at which $g$ is continuous. Take $b^{\prime}$ such that $0<g(b)-g\left(b^{\prime}\right)<f(a)-\lim _{i \rightarrow \infty} f\left(a_{i}\right)$. Let $X$ and $Y$ be random variables in $\mathbb{X}_{0}^{*}$ which satisfy $\sigma_{X}^{2}=b$ and $\sigma_{Y}^{2}=b^{\prime}$. Then, for all $i$,

$$
U\left(\mu_{Y+a_{i}}, \sigma_{Y+a_{i}}^{2}\right)=f\left(a_{i}\right)-g\left(b^{\prime}\right)<U\left(\mu_{X+a}, \sigma_{X+a}^{2}\right)=f(a)-g(b) .
$$

Since $U\left(\mu_{X+a}, \sigma_{X+a}^{2}\right)<U\left(\mu_{Y+a}, \sigma_{Y+a}^{2}\right)$, it follows from Axiom A6* that $X+a \sim Y+c$ for some $c<a$. However, $Y+a_{k} \prec X+a$ for some $c<a_{k}<a$. This is a contradiction. Hence $f$ has no gap, so is continuous on $\mathbb{R}$.

Suppose that $g$ has a gap at $a \in \mathbb{R}_{+}$. Let $\left\{a_{i}\right\}$ (respectively, $\left\{b_{i}\right\}$ ) be a strictly increasing (respectively, decreasing) sequence which converges to $a$ from below when $a>0$ (respectively, from above when $a \geq 0$ ). Assume that $\lim _{i \rightarrow \infty} g\left(b_{i}\right)>g(a)$. We derive a contradiction. When $\lim _{i \rightarrow \infty} g\left(a_{i}\right)<g(a)$, the proof is similar. Let $X$ and $X_{i}$ for $i$ be random variables in $\mathbb{X}_{0}^{*}$ such that $\sigma_{X}^{2}=a$ and $\sigma_{X_{i}}^{2}=b_{i}$. Since $f$ is continuous, take $b, b^{\prime} \in \mathbb{R}$ such that $0<f(b)-f\left(b^{\prime}\right)<\lim _{i \rightarrow \infty} g\left(b_{i}\right)-g(a)$. Then for all $i$,

$$
U\left(\mu_{X_{i}+b}, \sigma_{X_{i}+b}^{2}\right)=f(b)-g\left(b_{i}\right)<U\left(\mu_{X+b^{\prime}}, \sigma_{X+b^{\prime}}\right)=f\left(b^{\prime}\right)-g(a) .
$$

Since $U\left(\mu_{X_{1}+b}, \sigma_{X_{1}+b}^{2}\right)<U\left(\mu_{X+b^{\prime}}, \sigma_{X+b^{\prime}}\right)<U\left(\mu_{X+b}, \sigma_{X+b}\right)$, it follows from Axiom A3* that $X+b^{\prime} \sim\left\langle X+b ; \alpha ; X_{1}+b\right\rangle$ for some $0<\alpha<1$. Then we have 


$$
\begin{aligned}
U\left(\mu_{X+b^{\prime}}, \sigma_{X+b^{\prime}}\right) & =f(b)-g\left(\alpha \sigma_{X}^{2}+(1-\alpha) \sigma_{X_{1}}^{2}\right) \\
& =f(b)-g\left(\alpha a+(1-\alpha) b_{1}\right) \\
& <f(b)-g\left(b_{n}\right)(\text { for some } n) \\
& =U\left(\mu_{X_{n}+b}, \sigma_{X_{n}+b}^{2}\right) .
\end{aligned}
$$

This is a contradiction. Hence $g$ has no gap.

It remains to show that $f$ is unbounded. To show this, take any $X \in \mathbb{X}_{0}^{*}$ with $\sigma_{X}^{2}>0$, so $g\left(\sigma_{X}^{2}\right)>0$. By Axiom A7, we can define $\left\{a_{i}: i \in N\right\}$ by $a_{1} \sim X, a_{k+1} \sim X+a_{k}$ for $k=1,2, \ldots$. Then $f\left(a_{1}\right)=-g\left(\sigma_{X}^{2}\right)$ and $f\left(a_{k+1}\right)=f\left(a_{k}\right)+f\left(a_{1}\right)$ for $k \in N$. Hence $f\left(a_{n}\right)=n f\left(a_{1}\right)<0$ for all positive integers $n$. Hence $f$ is unbounded below. Similarly, we define $\left\{b_{i}: i \in N\right\}$ by $0 \sim X+b_{1}, b_{k} \sim X+b_{k+1}$ for $k \in N$. Then $f\left(b_{1}\right)-g\left(\sigma_{X}^{2}\right)=0$ and $f\left(b_{k}\right)=f\left(b_{k+1}\right)-g\left(\sigma_{X}^{2}\right)$ for $k \in N$. Hence $f\left(b_{n}\right)=n f\left(b_{1}\right)>0$ for all $n$, Hence $f$ is unbounded above. This completes the proof of (1). The uniqueness of $f$ and $g$ follows from Theorem 2 in Chapter 6 of [11].

(2) Necessity of Axiom B2 easily follows. Thus we show its sufficiency. Take any $X, Y, Z \in$ $\mathbb{X}_{c}^{*}$ for some $c \in \mathbb{R}$. By Axiom A6*,$X \sim Z+a$, and $Y \sim Z+b$ for some $a, b \in \mathbb{R}$. Then by Axiom B2, $X+b \sim Z+a+b$ and $Y+a \sim Z+b+a$. By transitivity of $\sim, X+b \sim Y+a$, so that Axiom B1 holds true.

Take any $a \in \mathbb{R}$ and any $X, Y \in \mathbb{X}_{0}^{*}$ with $\sigma_{X}^{2} \neq \sigma_{Y}^{2}$. By Axiom B6* ${ }^{*}, X+a \sim Y+c$ for some $c \in \mathbb{R}$. By Axiom B2, $X+a+x \sim Y+c+x$ for all $x \in \mathbb{R}$, so $U\left(a+x, \sigma_{X}^{2}\right)=U\left(c+x, \sigma_{Y}^{2}\right)$. Thus, (1) implies that

$$
f(a+x)-f(c+x)=g\left(\sigma_{X}^{2}\right)-g\left(\sigma_{Y}^{2}\right) \neq 0 .
$$

Since $\sigma_{X}^{2}, \sigma_{Y}^{2}$, and $x$ are arbitrary and $f$ and $g$ are continuous and strictly increasing, $f$ must be linear. The uniqueness of $g$ follows from the uniqueness in (1).

(3) It is easy to show necessity of Axiom B3. Thus we show its sufficiency. Since Axiom B3 implies Axiom B2, we have $U\left(\mu_{X}, \sigma_{X}^{2}\right)=\mu_{X}-g\left(\sigma_{X}^{2}\right)$ for all $X \in \mathbb{X}^{*}$. Take any $X \in \mathbb{X}_{0}^{*}$ and any $w \in \mathbb{R}$. Then, by Axiom A6, $X+w \sim a+w$ for some $a \in \mathbb{R}$. Thus $U(X+w)=U(a+w)$, which gives $g\left(\sigma_{X}^{2}\right)=-a$. By Axiom B3, $\lambda X+x \sim \lambda a+x$ for all $x \in \mathbb{R}$ and all $\lambda>0$, so that $g\left(\lambda^{2} \sigma_{X}^{2}\right)=-\lambda a$ for all $\lambda>0$. Since $X \in \mathbb{X}_{0}^{*}$ is arbitrary, we obtain that, for all $\lambda>0$ and all $x \geq 0$,

$$
\lambda g(x)=g\left(\lambda^{2} x^{2}\right) .
$$

Since $g$ is continuous, we must have $g(x)=\theta \sqrt{x}$ for a unique $\theta>0$.

(4) Necessity of Axiom B4 easily obtains. Thus we show its sufficiency. Assume that Axiom B4 holds. For $X \in \mathbb{X}^{*}$, we shall write $\sum_{n} X=(\cdots((X+X)+X) \cdots)+X$ (or simply $X+\cdots+X, n$ times in the sum). First, we show two claims. 
Claim 1. If $X \sim 0$, then $\sum_{n} X \sim 0$.

Proof. If $X+Y \sim Y$, then by Axiom B4, $X+X+Y \sim X+Y$, so $\sum_{2} X+Y \sim Y$. Thus applying Axiom B4 $n$ times, we obtain that $\sum_{n} X+Y \sim Y$. Letting $Y=0$, we obtain the desired result.

Claim 2. If $X \neq 0, Y \neq 0, X \sim 0$, and $Y \sim 0$, then $\mu_{X} / \mu_{Y}=\sigma_{X}^{2} / \sigma_{Y}^{2}$.

Proof. Take any $X, Y \in \mathbb{X}^{*}$ with $X \neq 0, Y \neq 0, X \sim 0$, and $Y \sim 0$. Suppose that $\mu_{X} / \mu_{Y}<\sigma_{X}^{2} / \sigma_{Y}^{2}$. Then there is a rational number $\frac{m}{n}>0$ such that $\mu_{X} / \mu_{Y}<\frac{m}{n}<\sigma_{X}^{2} / \sigma_{Y}^{2}$. Then $n \mu_{X}<m \mu_{Y}$ and $m \sigma_{Y}^{2}<n \sigma_{X}^{2}$, so that $U\left(n \mu_{X}, n \sigma_{X}^{2}\right)<U\left(m \mu_{Y}, m \sigma_{Y}^{2}\right)$. Thus

$$
U\left(\mu_{\sum_{n} X}, \sigma_{\sum_{n} X}^{2}\right)<U\left(\mu_{\sum_{m}}, \sigma_{\sum_{m} Y}^{2}\right)
$$

so that $\sum_{n} X \prec \sum_{m} Y$. But, Claim 1 implies that $0 \sim \sum_{n} X$ and $0 \sim \sum_{m} Y$, so $\sum_{n} X \sim$ $\sum_{m} Y$, a contradiction. When $\sigma_{X}^{2} / \sigma_{Y}^{2}<\mu_{X} / \mu_{Y}$, a similar contradiction obtains. Hence, $\mu_{X} / \mu_{Y}=\sigma_{X}^{2} / \sigma_{Y}^{2}$.

Fix $Z \in \mathbb{X}^{*}$ with $Z \sim 0$ and $\mu_{Z}>0$. Let $\theta=\mu_{Z} / \sigma_{Z}^{2}$. By Axiom A6, for any $X \in \mathbb{X}^{*}$, $X \sim c_{X}$ for some $c_{X} \in \mathbb{R}$. Then $\left(X-c_{X}\right)+c_{X} \sim c_{X}$, so by Axiom B4, $X-c_{X} \sim 0$. By Claim 2, $\sigma_{X}^{2} / \sigma_{Z}^{2}=\left(\mu_{X}-c_{X}\right) / \mu_{Z}$. Hence, $c_{X}=\mu_{X}-\theta \sigma_{X}^{2}$. Note that, for all $X, Y \in \mathbb{X}^{*}$, $X \precsim Y$ iff $c_{X} \leq c_{Y}$. Hence, $U\left(\mu_{X}, \sigma_{X}^{2}\right)=\phi\left(\mu_{X}-\theta \sigma_{X}^{2}\right)$ for a strictly increasing function $\phi$ on $\mathbb{R}$. The uniqueness of $\theta$ follows from Claim 2. This completes the proof.

\section{References}

1. R.E. Bailey, The Economics of Financial Markets. Cambridge University Press, 2005.

2. S. Barbera, P.J. Hammond, and C. Seidell, Handbook of Utility Theory, Volume 1: Principle, Kluwer Academic Press, 1998.

3. S. Barbera, P.J. Hammond, and C. Seidell, Handbook of Utility Theory, Volume 2: Extensions, Kluwer Academic Press, 2004.

4. D. Duffie, Security Markets, Academic Press, 1988.

5. L. Eeckhoudt, C. Gollier, and H. Schlesinger, Economic and Financial Decisions under Risk. Princeton University Press, 2005.

6. L.G. Epstein, Decreasing risk aversion and mean-variance analysis, Econometrica 53, 945-961, 1985.

7. P.H. Farquhar and Y. Nakamura, Constant exchange risk properties. Operations Research 35(2) 206-214, 1987. 
8. P.C. Fishburn, On the foundations of mean-variance analysis, Theory and Decision 10, 99-111, 1979.

9. P.C. Fishburn, Stochastic dominance and the foundations of mean-variance analyses, Research in Finance 2, 69-97, 1980.

10. P.C. Fishburn, The Foundations of Expected Utility. D.Reidel Publishing Company, Dordrecht, Holland, 1982.

11. D.H.Krantz, R.D. Luce, P. Suppes, and A. Tversky, Foundations of Measurement, Volume 1, Additive and Polynomial Representations. Academic Press, 1971.

12. A. Löffler, Variance aversion implies $\mu-\sigma^{2}$-criterion, Journal of Economic Theory 69, $532-539,1996$.

13. H. M. Markowitz, Portfolio selection. Journal of Finance 7(1), 77-91, 1952.

14. H. M. Markowitz, Portfolio Selection: Efficient Diversification of Investments. Yale University Press, 1959.

15. Y. Nakamura, Lexicographic quasilinear utility. Journal of Mathematical Economics $37,157-178,2002$.

16. A. Pollatsek and A. Tversky, A theory of risk, Journal of Mathematical Psychology 7, 540-55, 1970.

17. X. Qu, Subjective mean-variance preferences without expected utility, Working paper, 2015.

18. U. Safra and U. Segal, Constant risk aversion. Journal of Economic Theory 83, 19-42, 1998.

19. L.J. Savage, The Foundations of Statistics, Wiley: NewYork; 2nd revised edition, Dover, New York, 1954.

20. J. von Neumann and O. Morgenstern, Theory of Games and Economic Behavior. Princeton: Princeton University Press, 1944 (2nd edn., 1947; 3rd edn, 1953). 